\title{
Claves para pensar en la construcción de un objeto de investigación complejo: decisiones teórico- metodológicas en un abordaje multimétodo sobre niños/as, adolescentes y jóvenes que trabajan en las calles (La Plata, Buenos Aires, Argentina). ${ }^{1}$
}

Keys to think about building a complex object of research: theoretical and methodological decisions in a multi-method approach about children, adolescents and young people who work on the streets

\author{
María Eugenia Rausky \\ María Laura Crego \\ María Laura Peiró \\ Javier Alberto Santos \\ Universidad Nacional de La Plata \\ eugeniarausky@gmail.com(ARGENTINA)
}

Recibido: 29.092015

Aceptado: 16.03 .2016

\section{RESUMEN}

El artículo da cuenta de las decisiones teórico-metodológicas puestas en juego en una investigación empírica sobre niños/as, adolescentes y jóvenes que trabajan y/o viven en la calle en la ciudad de La Plata (capital de la Provincia de Buenos Aires, Argentina). La misma buscar reconocer la magnitud y particularidades del fenómeno en cuestión en dicha ciudad-en la que nunca se llevó a cabo una investigación de estas características- a través de un abordaje metodológico

\footnotetext{
${ }^{1}$ Este artículo se enmarca en los proyectos del equipo de investigación sobre Pobreza, Trabajo y Políticas Sociales en el Gran La Plata (con financiamiento del Programa Nacional de Incentivos Docentes y del PIP -Conicet 1673/12) y en el proyecto "Trabajo infantil y adolescente en espacios públicos de la ciudad de La Plata: un estudio de sus dimensiones y características" (financiado por la Agencia Nacional de Promoción Científica y Tecnológica. PICT código 2012-0026).
} 
sustentado en un diseño mixto de tipo secuencial. Esta presentación en particular, sitúa su eje de reflexión en la importancia de explicitar a la comunidad científica cómo se van articulando las decisiones teóricas y metodológicas en un proceso de investigación que aborda un objeto complejo.

El trabajo se organiza en tres apartados: en el primero se explicitan las preguntas que se formulan en este estudio y su relevancia; en el segundo se abordan los conceptos orientadores de la investigación -tanto teóricos como operativos-; en el tercero se da cuenta del conjunto de decisiones metodológicas consideradas a fin de llevar adelante el estudio, focalizando en lo realizado en la primera fase del trabajo de campo - un censo orientado a relevar a la totalidad de sujetos que integran las unidades de análisis-. Por último, en las conclusiones de destaca la importancia de brindar elementos que contribuyan a investigaciones que parten de intereses similares, como así también dar cuenta de las decisiones puestas en juego para la construcción y abordaje de un objeto de indagación complejo.

\title{
PALABRAS CLAVE
}

Métodos mixtos; Trabajo; Niñez; Juventud; Espacio público.

\begin{abstract}
The paper covers theoretical and methodological decisions playing their role in an empirical research on children, adolescents and young people who work and/or live in the streets in La Plata City (capital of the Province of Buenos Aires, Argentina). This research aims to recognize both magnitude and peculiarities of the phenomenon in said city- where a research of said characteristics has not been made before- via a methodological approach backed by a sequential type mixed design. This presentation, particularly, focus its axis in thinking on the importance to make it explicit to the scientific community the means and ways theoretical and methodological decisions become articulated in a research process which approaches a complex object.

The work is organized in three sections. The first one deals with explicitations and questions being made in this research and their relevance; the second section is about concepts that orientate research; in the third one an approach is made to the group of methodological decisions considered in order to carry out the study, focusing in what has been made in the first fieldwork stage-a poll aiming to relevate all of the subjects that integrate the analysis-. At last, in the conclusions offered it is outlined the importance of producing elements contributing to researches starting from similar interests, as well as covering decisions playing their role to construct and approach a complex object of questioning
\end{abstract}




\section{KEY WORDS}

Mixed Methods; Labour; Childhood; Youth; Public Space.

\section{INTRODUCCIÓN}

La construcción de objetos de conocimiento en el campo de la investigación científica no se da en el vacío, sino que debe reconocer y dialogar con el acervo de conocimientos previos sobre el fenómeno en cuestión. Asumir esa tarea es un gran desafío, sobre todo si se tiene como horizonte el aporte de nuevo conocimiento a un campo complejo como el de los niños/as, adolescentes y jóvenes que trabajan y/o viven en la calle. Hacemos mención a esta categoría amplia -trabajar y/o vivir en la calle- ya que si bien el foco de la investigación está puesto en relevar la cuestión laboral, en muchos casos ambas situaciones se solapan: aquellos que trabajan en la calle a veces de manera transitoria duermen en el espacio público y quienes viven en éste con regularidad, suelen desplegar estrategias laborales en el mismo espacio para sobrevivir.

Sumergidos en esta temática, el artículo tiene como objetivo dar cuenta del conjunto de decisiones teórico-metodológicas puestas en juego en el marco de una investigación empírica sobre niños, adolescentes y jóvenes que trabajan y/o o viven en la calle en el ámbito de la ciudad de La Plata (capital de la Provincia de Buenos Aires, Argentina). Se entiende que la explicitación de tales decisiones configura un ejercicio que aporta dos insumos al campo en el que se inscribe este estudio: 1) demuestra el modo en que se construye sociológicamente el abordaje de un objeto de investigación complejo: desde la formulación inicial de las preguntas, su justificación y la delimitación de las definiciones teóricas y operativas, y 2) aporta un cúmulo de decisiones metodológicas en el contexto de un abordaje mixto centradas en el cómo, dónde, cuándo, con quiénes, etc., que puede ser una referencia de utilidad para quienes procuren desarrollar una investigación de similares características.

El estudio de las personas que viven y/o trabajan en la calle ha sido extensamente abordado por las ciencias sociales. La necesidad de explorar las particularidades que asume esta figura paradigmática de la exclusión social ha llevado a conocer sus características -quiénes son, qué estrategias despliegan para sobrevivir, qué lazos construyen con las instituciones, etc.-, como así también intentar develar los procesos que conducen a dicha situación. Algunas investigaciones han hecho foco en el análisis del rol que han tenido los determinantes sociales en la configuración de estos procesos, mientras que otras han enfatizado en los factores individuales (Paugam, 2007).

No hay dudas respecto de que este grupo de personas ha representado y representa un objeto de interés socio-antropológico con múltiples posibilidades de exploración. Ahora bien, ese gran grupo de personas que residen y/o trabajan en la calle no ha sido abordado como un todo homogéneo. Habitualmente los estudios han distinguido modalidades de aproximación a este objeto con 
distintos recortes: por un lado, el estudio de los llamados homeless (para los angloparlantes), los sans domicile fixe (para los francófonos) y las personas sin techo o en situación de calle (para el habla española), en tanto sujetos que no cuentan con una residencia fija y adecuada para dormir, o que duermen en albergues o instituciones, y, por otro lado, el estudio de las personas que utilizan la calle como lugar de trabajo, sin residir allí. Aunque en efecto, entre uno y otro grupo hay un continuum de situaciones intermedias, generalmente los abordajes se han centrado en el estudio diferenciado de ambas poblaciones.

A este recorte hay que sumarle uno adicional: el estudio específico por grupo etario, configurándose así un conjunto de investigaciones interesadas en explorar la situación de niños y jóvenes en particular. Se han registrado iniciativas en diferentes urbes del mundo tendientes tanto a cuantificar el fenómeno -a través de encuestas- como a indagarlo desde perspectivas cualitativas. Revisando los antecedentes en Argentina se encuentran distintas tendencias al respecto. En el caso de los relevamientos sobre trabajo infantil y juvenil las estrategias de recopilación de la información descansan principalmente en encuestas a hogares -con el subregistro que acarrea- , mientras que de las pocas experiencias sistematizadas y publicadas que han procurado contabilizar el volumen de chicos que se encuentran en la calle trabajando y/o viviendo, solamente un estudio ha desarrollado un censo in situ en la ciudad de Córdoba -Capital de la Provincia de Córdoba- (Peralta et. al, 2011), el resto de los trabajos se han basado en el conteo y caracterización a través del ingreso de dichos sujetos a instituciones destinados a atenderlos Pojomovski (2008).

En general, uno de los aspectos pendientes que suelen tener tales estudios -al menos en Argentina- es el de la explicitación y puesta en juego de las decisiones metodológicas que han guiado las investigaciones empíricas, así, mucho se conoce sobre resultados y poco sobre los modos en que se han delimitado los objetos de investigación y los métodos y técnicas que se han utilizado para arribar a ellos. En este sentido, este artículo propone recuperar el conjunto de decisiones teórico-metodológicas adoptadas para estudiar al colectivo de niños/ as, adolescentes y jóvenes que trabajan y/o viven en la calle.

La estructura expositiva del texto descansa en cuatro apartados. En el primero se presentan las preguntas que guían la investigación empírica, la justificación del caso de estudio, destacándose algunas de sus particularidades. En el segundo se recuperan los conceptos centrales y las definiciones operativas construidas para esta investigación. En el tercero se explicitan las decisiones metodológicas: estrategias de abordaje, técnicas de producción de la información, decisiones de muestreo y aspectos operativos relativos al trabajo de campo. El artículo concluye con un apartado de reflexiones finales. 


\section{EL PUNTO DE PARTIDA}

El estudio que el equipo está llevando a cabo apunta a reconocer la magnitud y particularidades del fenómeno en cuestión en el ámbito de la ciudad de La Plata -en la que nunca se llevó a cabo una investigación de estas característicasa través de una propuesta metodológica de carácter mixto secuencial (Teddlie y Tashakori, 2005). Las preguntas orientadoras buscan delimitar ¿cuántos son?, ¿qué hacen?, ¿con quiénes?, ¿cómo llegaron allí?, ¿cuál es su origen social?, ¿qué vínculos tienen con sus familias?, ¿y con las instituciones estatales y no estatales? ¿Con los habitantes de la ciudad?, ¿qué usos hacen del espacio público?, ¿qué circuitos transitan?, ¿cómo vivencian su experiencia con el trabajo?, ¿cómo impacta en ellos el hecho de que su trabajo se desarrolle en un ámbito público como la calle?, ¿qué diferencias se presentan entre niños/as, adolescentes y jóvenes y fundamentalmente entre aquellos que sólo trabajan en la calle y quienes trabajan y habitan en ella?

Según los datos del Censo Nacional de Población y Vivienda, el Partido de La Plata (compuesto por el casco urbano fundacional de la ciudad y las comunas circundantes) tenía en 2010 un total de 654.324 habitantes. Los niños, adolescentes y jóvenes representaban casi el $40 \%$ de la población total del partido (20,6\% niños de 0 a 13 años, 9,4\% adolescentes de 14 a 19 años y 9,4\% jóvenes de 20 a 24 años). Según las proyecciones realizadas por el Instituto Nacional de Estadísticas y Censos, se estima que su población total se incrementó en un 3,3\% entre 2010 y 2014 -fecha de realización del relevamiento-.

Como ya se adelantó, la pregunta sistemática en torno a los chicos que trabajan y/o viven en las calles no es en absoluto novedosa ${ }^{2}$. Hace casi 20 años atrás, Rizzini (1996) sistematizaba los estudios que al respecto se habían producido en América Latina y advertía que fue en la década del 80 cuando se detectó claramente un progresivo interés en el estudio de este tema. A partir de entonces y hasta la actualidad pueden encontrarse numerosos estudios abocados a conocer las especificidades que asume este fenómeno urbano en distintos países latinoamericanos (Hugginis y Rodríguez, 2004; ScheperHughes y Hoffman, 1999; Lucchini, 1996; Hetch, 1998; OIT, 2011; OIT, 2002; Unicef, 2000; Pojomovsky, 2008, Makowski, 2010; Makowski, et. al. 2010; Rial, Rodriguez y Vomero, s/f; García Silva, 2014, entre muchos otros). Paralelamente, desde hace dos décadas el Instituto de Formación para Educadores de Jóvenes, Adolescentes y Niños Trabajadores de América Latina y El Caribe (IFEJANT), a través de sus órganos de difusión como la Revista Niños, Niñas y Adolescentes Trabajadores del Sur (NATs) y su editorial que

${ }^{2}$ Cabe señalar que es más profusa la literatura que aborda el fenómeno de los chicos que viven en la calle (en las cuales se analiza el trabajo que se desarrolla en la calle como una dimensión más en la vida de estos chicos), que aquella que estudia específicamente el trabajo callejero, por ello aquí se retomarán parte de esas investigaciones en la medida en que constituyen los antecedentes relevantes para este campo de estudio.. 
lleva publicados cientos de libros, han difundido numerosos estudios y ensayos sobre trabajo infantil, entre los que se encuentran algunas reflexiones referidas a la niñez que trabaja en las calles, desde una perspectiva que aboga por el reconocimiento de las prácticas laborales en la infancia.

Ahora bien ¿por qué el interrogante por los chicos que trabajan y/o viven en las calles sigue despertando interés en el marco de las ciencias sociales? ¿Presenta alguna particularidad en el contexto actual?

En el caso de los niños/as y adolescentes, el avance en materia de derechos con el que esta población ha sido protegida es uno de los pivotes más directos para problematizar la inserción temprana en el mundo del trabajo ${ }^{3}$ : infancia y trabajo se piensan como instancias incompatibles jurídicamente. De hecho, en el contexto argentino la progresiva reducción del trabajo infantil ha sido uno de los objetivos explícitos en materia de política pública.

La situación de los adolescentes amerita otra lectura ya que en términos jurídicos, la población comprendida entre los 16 y 17 años de edad, si bien tiene permitido trabajar, lo puede hacer en la medida en que se garanticen una serie de protecciones -referidas a la cantidad de horas de trabajo semanales, a la prohibición del trabajo nocturno y al no desempeño en actividades que impliquen tareas peligrosas/riesgosas-.

Por su parte, en el caso de los jóvenes a partir de los 18 años lo que se problematiza no es su incorporación al mundo del trabajo - puesto que de hecho se habilita jurídicamente su inserción laboral- sino más bien el desempleo o la inserción precaria (Peiró y Rausky, 2009). Aún en contextos de crecimiento y mejora de los niveles de empleo y de las condiciones de trabajo generales, los jóvenes constituyen -al igual que en la mayoría de los países- uno de los segmentos más vulnerables del mercado laboral, presentando tasas de desempleo mayores y condiciones de trabajo más precarizadas que los adultos.

Trazado el mapa de la situación de la población que aquí interesa estudiar caben las siguientes acotaciones:

a) que en términos jurídicos no puede equipararse el trabajo desarrollado por niños/as, adolescentes y jóvenes ya que uno está prohibido, el otro protegido y el último permitido;

b) que las expectativas sociales generadas en torno a estos grupos de edad tampoco son idénticas. La cronologización de la vida característica de las sociedades occidentales (Groppo, 2000) en donde los límites entre la infancia, la juventud y la adultez están social y culturalmente demarcados, hacen que las expectativas asignadas a los sujetos de acuerdo a los grupos de edad de pertenencia sean diferentes, de allí que no se pueda tratar homólogamente a los niños/as, adolescentes y jóvenes.

${ }^{3}$ Además de la sanción en 2005 de la Ley Nacional № 26.061 de Protección Integral de los Derechos de las Niñas, Niños y Adolescentes (que adopta los lineamientos de la Convención Internacional de los Derechos del Niño), en 2008 se promulgó la Ley Nacional № 26.390 sobre Prohibición del Trabajo Infantil y Protección del Trabajo Adolescente, destinada a elevar progresivamente la edad mínima de admisión al empleo -16 años- y regular el trabajo de los adolescentes.. 
Si bien los aspectos arriba descriptos llevan a advertir sobre la necesidad de no construir una categoría homogénea para describir las prácticas laborales de estos sujetos, para el caso del fenómeno del trabajo callejero sí hay un rasgo común a destacar y es que todos comparten una misma condición: el hacer uso del espacio público para desplegar actividades laborales, el cual de acuerdo a lo estipulado por el código del Espacio Público vigente en la ciudad de La Plata (Ordenanza $\mathrm{N}^{\mathrm{o}}$ 9.880), dispone que gran parte de las actividades desarrolladas por estos niños/as, adolescentes y jóvenes se tipifiquen como prohibidas. Traer a colación esta cuestión resulta de central importancia por los efectos que tiene en las personas que utilizan las calles para trabajar, puesto que generalmente la calle siempre fue, y continúa siendo, un lugar ocupado por los más destituidos, que permanecen en ellas en búsqueda de algún beneficio (Gregori, 2000). Como sugieren Scheper-Hughes y Hoffman (1999) el término "chicos de la calle" insinúa que los niños/as y adolescentes están ocupando ilegítimamente el espacio público, que algo está fuera de lugar. De ello da cuenta por ejemplo la persecución policial, municipal, y hasta incluso de los propios ciudadanos para con quienes están trabajando en la calle. Existe una representación difundida en todo el cuerpo social que considera que la calle contamina moralmente.

En el estudio que el equipo desarrolla, lejos de aproximaciones de carácter moral que buscan identificar a la calle como un espacio no apropiado para los chicos, interesa recuperar las condiciones y los modos de trabajo y de vida de estos sujetos, viendo, en línea con lo que tempranamente planteaba Hetch (1998) por qué la calle representa una opción y qué brinda comparativamente frente a otros espacios.

Habitualmente quienes se abocan al estudio de este fenómeno circunscriben el análisis a aquel núcleo duro de sujetos que se encuentran en situaciones límite, es decir, aquellos niños/as, adolescentes y jóvenes provenientes de sectores sociales postergados que encuentran en la calle su único refugio para la sobrevivencia. Si bien este grupo es el que más notoriedad y presencia tiene en el espacio público, interesa destacar que allí también se reúnen sujetos que provienen de otros sectores sociales y en los que se condensan otras problemáticas que no son únicamente la exclusión social y la pobreza. En este sentido, se entiende que la calle en tanto espacio de trabajo es habitada por múltiples actores que pueden distinguirse apelando al menos a dos criterios: el grupo de edad (niños/as, adolescentes, jóvenes y adultos) y/o el sector social de procedencia (desde los que se hallan en los márgenes hasta quienes provienen de las clases medias). En general, en los ámbitos urbanos como el que se estudia, tales criterios se combinan de manera particular: los niños/as y adolescentes que llegan a desarrollar actividades laborales en las calles (acompañados por adultos o no) suelen provenir de sectores pobres, representando la calle una de las alternativas "al alcance" para la generación de ingresos; en cambio, esta relación no es tan directa para el caso de los jóvenes, ya que en la calle se mezclan jóvenes pobres desarrollando actividades de subsistencia, con jóvenes de sectores medios o medios-bajos realizando actividades más vinculadas a la expresión artística, a la vida bohemia o al "rebusque" temporario. Para el caso de 
los trabajadores migrantes -en la ciudad hay un grupo importante de trabajadores callejeros senegaleses-, entran en juego además otros factores como la condición de regularidad/irregularidad de su documentación, el tiempo de residencia, la posibilidad de establecer redes, etc.

En esta investigación interesa hacer foco justamente en esa heterogeneidad, de modo tal que se pueda analizar la calle como un espacio en el que se pueden encontrar trabajadores en diferentes situaciones: desde las más extremas y dramáticas como lo puede representar el caso de los que trabajan y viven allí, para quienes pareciera no haber otra opción que la calle, hasta aquellas en que los trabajadores pueden elegirla frente a otras posibilidades.

\section{EL RECORTE CONCEPTUAL}

Teniendo la certeza de que la explicitación de las decisiones teóricas y metodológicas de una investigación ayuda a comprender más cabalmente la lógica con la que se construye el objeto de estudio y los datos que de él se derivan, en este apartado se presentan los conceptos centrales de la investigación y las definiciones operativas construidas luego de una extensa revisión bibliográfica de los campos que entrecruzan este objeto de estudio.

¿Chicos en/de la calle?

Hasta no hace mucho tiempo la literatura interesada en abordar el fenómeno de los chicos y chicas que se encuentran en la calle se hallaba permeada por una distinción prácticamente incuestionable: los chicos "en" o "de" la calle. Tal diferenciación asume la existencia de dos amplios perfiles, considerando como uno de los factores centrales de dicha diferenciación la existencia o no de lazos familiares. En el primer perfil se incluía a quienes permanecen en la calle en busca de actividades generadoras de recursos, pero que retornan regularmente a sus hogares. El segundo perfil refería en cambio a aquellos que habitan de manera estable el espacio público y que tienen escaso o nulo contacto con sus familias (Rizzini, Caldeira, Ribeiro y Carvano, 2010).

Mientras que esta clasificación, que ha servido como aproximación al fenómeno, aún es sumamente pregnante tanto en el contexto de las instituciones que atienden a esta población como en el campo de las ciencias sociales, han surgido una serie de trabajos que la cuestionan, tal es el caso de los estudios de Hetch (1998), Aptekar y Abebe (1997), Dallape (1996) y Droz (2006), entre otros. Incluso, hay investigaciones como la de Makowski (2010) que sin proponerse cuestionar esta clasificación, introduce la interesante idea de pensar en las figuras de la itinerancia urbana -entendida como modalidad de experiencia social- que pueden encontrarse en las ciudades.

La distinción "de" o "en" acarrea una serie de desafíos tanto teóricos (cuál es el alcance y pertinencia de estos conceptos) como operativos (¿cuándo se es efectivamente un chico "de” o "en” la calle? ¿El límite entre dormir y no dormir en el espacio público basta como criterio demarcador?). Reconociendo las diferentes críticas desarrolladas y atentos al señalamiento de sus limitaciones, 
en esta investigación se recupera esta distinción a los fines de pensar aspectos característicos del campo y tomar en cuenta los interrogantes que plantea, aclarando entonces que el eje de atención será reconocer el trabajo que niños/as, adolescentes y jóvenes desarrollan en las calles, para dar lugar a la identificación de las múltiples formas de estar en la calle trabajando. Se asume que ubicar a los sujetos en función de la categoría clasificatoria "de/en" implica fundirlos en una homogeneidad que obstaculiza una buena descripción sociológica.

\subsection{Trabajo infantil, adolescente y juvenil}

El hecho de estudiar grupos etarios distintos como niños/as, adolescentes y jóvenes plantea la necesidad de diferenciar su situación jurídica: como se detalló, el niño se encuentra jurídicamente amparado frente al trabajo, el adolescente protegido y el joven habilitado para insertarse en el mundo laboral. De acuerdo con ello las definiciones sobre trabajo infantil, adolescente y juvenil fueron delimitadas en nuestra investigación por dos criterios: uno referido a la edad cronológica -criterio que se ajusta a las normativas vigentes-, el otro referido al tipo de actividades que se contemplan.

En el primer caso se considera trabajo infantil a aquel desarrollado por quienes tienen menos de 16 años de edad, trabajo adolescente al que se lleva a cabo por quienes tienen entre 16 y 17 años, y trabajo juvenil a aquel que se realiza por quienes integran la franja que va de los 18 a los 24 años. La delimitación de este criterio etario es arbitrario, tanto en lo referido a la población joven porque pone el techo en los 24 años, sin discutir la extensión ni las fronteras de lo que se puede considerar -social y/o subjetivamente- la etapa juvenil, como en lo que respecta a la definición de la población adolescente; sin embargo se lo considera de esta forma en nuestro estudio con el fin de poder contar con información de referencia (estadísticas públicas) y porque la delimitación dada por la legislación plantea situaciones diferenciales para quienes efectivamente se vuelcan a desarrollar actividades laborales a edades tempranas.

Una cuestión similar se suscita al definir el límite de edad inferior para el caso del trabajo infantil, límite que las estadísticas nacionales e internacionales suelen ubicar en los 5 años de edad. Respecto de esto último, por ejemplo, un censo sobre trabajo infantil callejero llevado a cabo en la ciudad de México considera en el conteo a los niños/as cuya edad es inferior a los 5 años y a los sujetos que los acompañan (UNICEF, 2000). Esta apuesta desarrollada por dicha investigación llevó a considerar la posibilidad de incluir estos casos en nuestro conteo.

En el segundo caso se asume que si de lo que se trata es de captar actividades laborales que se desarrollan en la vía pública, en principio se requiere de un encuadre lo suficientemente amplio como para visibilizar la variabilidad de las mismas, es decir que siguiendo a De la Garza Toledo (2001) y Boufartique (1996) se toma distancia de aquellas perspectivas que hacen un uso restringido 
del concepto de trabajo asociándolo directamente a la condición salarial, para dar lugar a una mirada capaz de integrar la amplitud característica en esta clase de inserciones, por cierto informales y precarias. Así, se contemplaron todas las actividades generadoras de ingresos (monetarios o no monetarios) ligadas a la producción de bienes, servicios y el limosneo. Fueron excluidas la prostitución y el delito ${ }^{4}$.

Una operacionalización inspirada en el reconocimiento de las características del campo y en los propios intereses de la investigación, llevó entonces a incluir en la variable trabajo a todas aquellas actividades informales que se desarrollan en la vía pública. Esto comprende a vendedores ambulantes; manteros (quienes fijan un puesto informal y temporario para la venta de distintas clases de productos); ciudacoches/lavacoches; limpiavidrios; lustrabotas; malabaristas y demás artistas callejeros; carreros; changarines de jardinería; y por último a quienes mendigan. Si bien cabe destacar que hay otras tantas actividades que se llevan a cabo en la calle y bajo condiciones de precariedad, como lo puede ser la labor de los canillitas $^{5}$, de los vendedores de comidas elaboradas instalados en puestos habilitados por el municipio o los feriantes, no son incluidos en esta definición por considerar que su trabajo se desarrolla bajo alguna relación de dependencia formalizada o bajo algún tipo de regulación municipal, acarreando otro tipo de características.

\subsection{El espacio público, la ciudad y la calle}

La teorización sobre el concepto de espacio tiene una importante y extensa trayectoria en el campo de la geografía, y un acotado -aunque interesante- lugar

${ }^{4}$ La primera por tratarse, en el caso de los niños/as y adolescentes, de violaciones de los derechos humanos y delitos de lesa humanidad, entendiendo que en estas actividades no hay proceso de trabajo alguno dado que la relación se establece por vía de la coerción y tiene un profundo significado de degradación del ser humano (Kohen, 2004); el segundo por considerar que estaríamos entrando en otro orden de problemas sociológicos. Incluso quienes se dedican a estudiar sociológicamente el delito entienden que definirlo es en sí mismo problemático, obligándonos a entrar en el complejo terreno de las tipologías, referidas a las acciones que son consideradas como tales, como a la clase de actores que las cometen, al margen de tomar en cuenta además el inevitable problema del relativismo cultural trasladado a la consideración del delito (Míguez, 2008). Se coincide en remarcar que el delito es la parte visible de otros procesos menos evidentes y de más vasto alcance (Kessler, 2006), que si bien en algunos aspectos pueden ser los mismos que subyacen al problema del trabajo callejero, también tiene sus dinámicas específicas.

Si bien se asume que tanto una como otra son actividades generadoras de recursos (y que los mismos actores pueden combinar temporalmente alguna de ellas con otro tipo de trabajo en la calle), requieren para su abordaje un conjunto de interrogantes y dispositivos particulares ligados a su especificidad. No se trata tanto de plantear dificultades metodológicas ligadas a la accesibilidad (por creerlos fenómenos impenetrables), pero sí por reconocerlos como objetos que requieren para su aproximación de otra clase de preguntas. No obstante ello, tal decisión no excluye que entre los interrogantes no se contemplen esta clase de actividades, sólo que ni serán analizadas en tanto trabajo, ni tampoco serán estudiadas en su cabal profundidad tal como lo haría cualquier especialista en temas de delito y aproximaciones a la prostitución en chicos/as

5 Vendedores de diarios y revistas en puestos habilitados. 
en la teoría sociológica. En este último caso los trabajos de Goffman (2006) en los que se evidencia una preocupación por el ordenamiento espacio-temporal de la actividad social y de Giddens (1998), quien recupera la importancia de los desarrollos que la geografía hizo en torno al concepto de espacio, no dejan de ser referencias centrales en nuestro campo.

Pérez Álvarez (2003) subraya que en la modernidad el espacio público ha tendido a ser conceptualizado tanto como concepto jurídico (publico vs. privado), como concepto socio-cultural (lugar para relacionarse); en este último sentido es que puede pensarse que en las ciudades los comportamientos de sus habitantes pueden crear y recrear espacios públicos que jurídicamente no lo son, es decir que lo que define su naturaleza no es su estatuto jurídico sino su uso. El concepto de espacio público tiene una importancia significativa para lo urbano y la ciudad porque es allí donde se manifiesta con más fuerza la crisis que la ciudad vive. Si bien el eje de indagación de este estudio se centra en el fenómeno del trabajo y no en los procesos de producción de la ciudad y lo urbano, se adhiere a la idea de considerar la dimensión espacial del trabajo (Lindón, 1997), ya sea como espacio en el cual se realiza el trabajo o como significados del espacio de trabajo, en tanto que son parte del fenómeno laboral en sí mismo. Como argumenta Lindón (2002) la renovación del concepto de trabajo y de los enfoques laborales de manera amplia, bien podría ser planteada a partir de la inclusión y problematización de su espacialidad, dimensión muchas veces olvidada en este campo del conocimiento.

En el caso de América Latina, mientras que algunas investigaciones sobre este colectivo han tomado al espacio público y a la calle como un dato (Invernizzi, 2003; Pérez López, s/f; Huggings y Rodriguez, 2004; Unicef, 2000; Cerquiera Filho y Neder; 2001), otras sí se han ocupado de problematizarla y de indagar los vínculos que los sujetos tejen con ella (Pojomovsky, 2008; Lucchini, 1996; Perez Álvarez, 2003; Makowski 2010; Leyra 2009; Rial, Rodriguez y Vomero, s/f). En línea con este último grupo de estudios, se recuperaron como ejes de observación y exploración del trabajo de campo algunas de las aproximaciones en las que estas investigaciones han incursionado para analizar la compleja y rica trama de relaciones que se establece entre los sujetos que trabajan, el espacio en el que se desarrolla su actividad y los otros actores que se encuentran allí.

Por ejemplo Makowski (2010) incorpora una serie de conceptos con un potencial heurístico que entendemos vale la pena recuperar. La itinerancia urbana entendida como "modalidad de experiencia social que espacializa las significaciones simbólicas de la exclusión; traduce en el espacio las continuidades y discontinuidades de la experiencia y de las relaciones sociales (...). Como forma narrativa de exclusión social, moldea y organiza el tiempo y el espacio: los espacios se vuelven móviles y los tiempos son siempre inestables" (Ibid: 135). Se reconocen al menos tres sentidos asociados a ella: 1) la ruptura -la salida a la calle como quiebre de la permanencia en el hogar-; 2) la repetición -búsqueda permanente de un lugar, de un anclaje-; 3) la trayectoria -en tanto fase final de la carrera de la calle-; al tiempo que adquiere una morfología, 
ya que hay lógicas de desplazamiento que cristalizan en: i) recorridos -que espacializan la rutina cotidiana, acotando los espacios-; ii) circuitos -que dan cuenta de los lugares e instituciones por las que transitan, y iii) ciclos -signados por aspectos climáticos y festividades-.

Pérez Álvarez (2003) focaliza la atención en los distintos usos que los chicos hacen de la ciudad, planteando la necesidad de pensar en dinámicas diferenciales, que llevan a la construcción de vínculos disímiles entre sus habitantes. A lo largo de su análisis emplea una operacionalización de la expresión territorialidad -categoría que incluye aspectos materiales, simbólicos e ideológicos-, en tres ámbitos: el espacio vivenciado -prácticas, rutinas y usos que los niños y jóvenes trabajadores hacen del espacio-; el espacio concebido -significados, representaciones e imágenes que dotan de afecto a los lugares y el espacio controlado o institucionalizado -costumbres, normas y acciones concretas reproducidas y expresadas en técnicas de organización y control instituidas por los propios niños y jóvenes que trabajan en sus territorios.

Creemos que estos modos de pensar los vínculos que los sujetos tejen con la calle nos aportan indicios interesantes para analizar la compleja y rica trama de relaciones que se establece entre los sujetos que trabajan, el espacio en el que se desarrolla su actividad y los otros actores que se encuentran en ese espacio.

Por último, cabe consignar que en términos operativos en este estudio se definió al espacio público como aquellos lugares "a cielo abierto" tales como calles, avenidas, diagonales, plazas, parques, plazoletas, rotondas y ramblas, incluyendo el segmento de edificios públicos en el margen que comprende la línea municipal y las paredes que delimitan su construcción, y excluyendo el interior de edificios públicos como ministerios, facultades, estación de trenes, ómnibus, transporte público, etc. En la fase cualitativa del trabajo se exploran en profundidad los modos en que los sujetos practican y vivencian este espacio recuperando los aportes de los autores mencionados.

\section{LAS DECISIONES METODOLÓGICAS: LA OPCIÓN POR UN ABORDAJE MIXTO}

El trabajo, de carácter exploratorio, ha sido realizado en dos servicios de iEl objeto de conocimiento operativizado en el conjunto de interrogantes planteados anteriormente, en diálogo con lo ya conocido del campo observacional, puso en evidencia la necesidad de pensar la articulación metodológica como vía necesaria para responder a cada uno de ellos de manera plena y articulada. Se optó por un diseño mixto secuencial en dos fases (Teddlie y Tashakori, 2006) para el abordaje in situ del trabajo en el espacio público de los niños/as, adolescentes y jóvenes.

En lo que sigue se reseñan las decisiones de abordaje, selección y recolección efectuadas en cada etapa del trabajo de campo. Este recorrido se encuentra en permanente diálogo con una revisión crítica de los planteos expresados al respecto y de los diseños utilizados en investigaciones 
latinoamericanas (UNICEF, 2000), europeas -en especial las francesas (Firdion, Marpsat y Mauger, 2000; Marpsat y Firdion, 2001; Marpsat, Firdion y Meron, 2000, Marpsat y Firdion 1998, Marpsat, 2008a y Marpsat 2008b)- y norteamericanas (Burt, et. al, 1999; Pergamit et. al, 2013), dedicadas al estudio de las personas que viven en la calle y, especialmente, de las orientadas al estudio de los niños/as, adolescentes y jóvenes que trabajan y/o viven en la calle.

El diseño mixto secuencial -que integra tanto estrategias cuantitativas como cualitativas- remite a que en la propuesta de indagación se abordan distintas fases (dos) en donde las estrategias metodológicas se integran. Es importante resaltar que cada fase es entendida como una unidad en donde se articulan instancias de conceptualización (decisiones en torno al qué objetivos, preguntas de investigación-), metodológicas (decisiones de selección, recolección y análisis) y de inferencia (asociadas con las explicaciones, comprensiones, descripciones que incluye a la teoría emergiendo, explicaciones e inferencias). Así, la propuesta de nuestra investigación se reconoce como multifase en tanto aborda más de una fase (en específico se trata de dos fases) e instancias articuladas en donde los procedimientos y técnicas de producción de información de corte cualitativo y cuantitativo entran en integración. El tipo de implementación de la propuesta es secuencial y esto implica asumir que los procedimientos metodológicos de la investigación no se darán concurrentemente sino de manera sucesiva en cada fase. En este sentido, la fase siguiente emerge de la anterior y es retroalimentada por ésta. De este modo, la investigación se desarrolla en dos fases: la primera, de tipo cuali-cuantitativa con énfasis cuantitativo (CUAL/CUAN+), y la segunda cuanti-cualitativa con énfasis cualitativo (CUAN/CUAL+). 


\begin{tabular}{|c|c|c|c|c|}
\hline \multicolumn{2}{|r|}{ 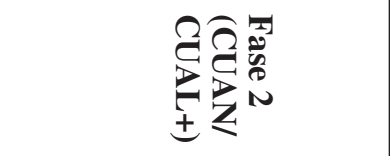 } & \multicolumn{2}{|r|}{ 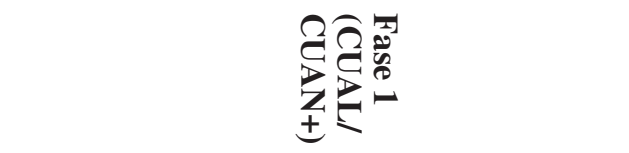 } & \multirow{2}{*}{$\begin{array}{l}\overrightarrow{0} \\
\overrightarrow{0} \\
\overrightarrow{0}\end{array}$} \\
\hline 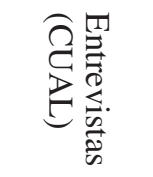 & 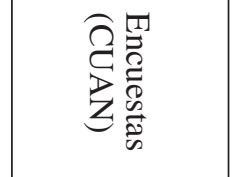 & 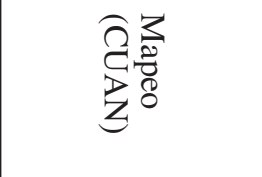 & 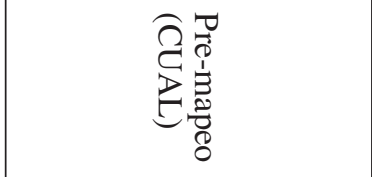 & \\
\hline 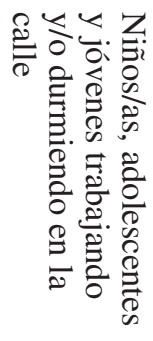 & 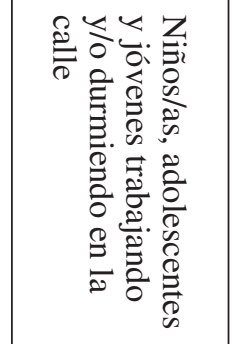 & 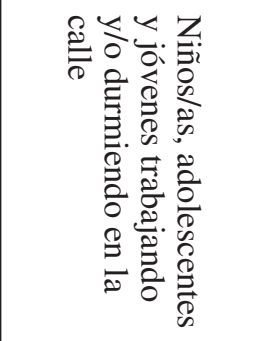 & 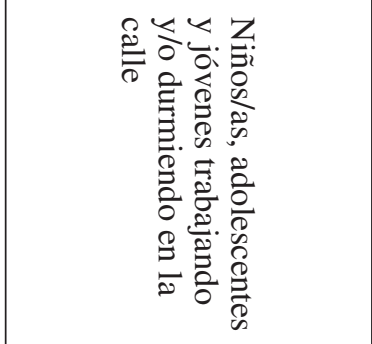 & 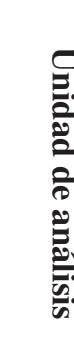 \\
\hline 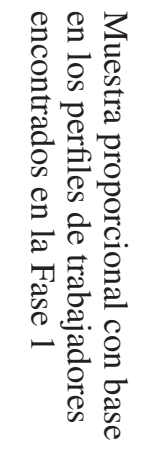 & 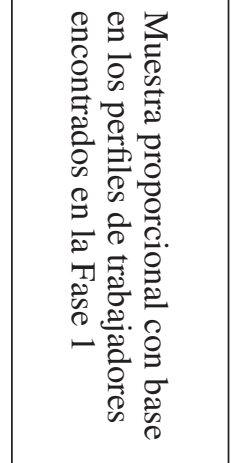 & $\S_{8}^{8}$ & 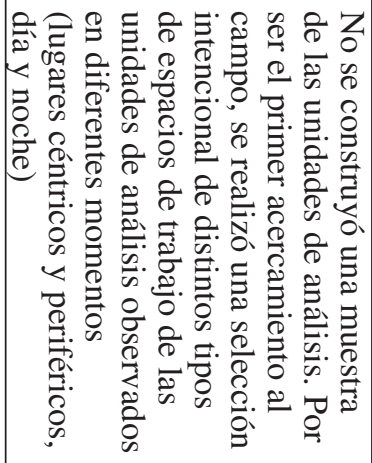 & है \\
\hline 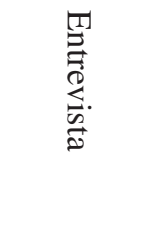 & 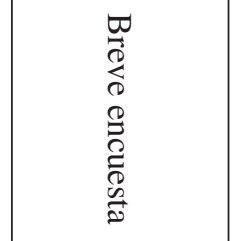 & $\begin{array}{l}\frac{T}{2} \\
\stackrel{2}{D} \\
\stackrel{2}{2} \\
\stackrel{2}{2}\end{array}$ & 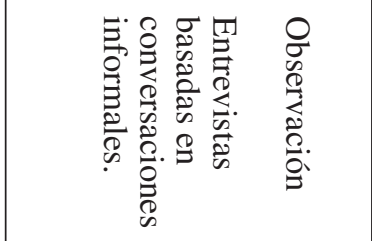 & อุำ \\
\hline 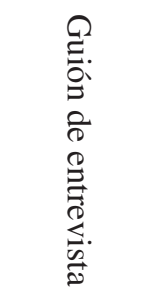 & 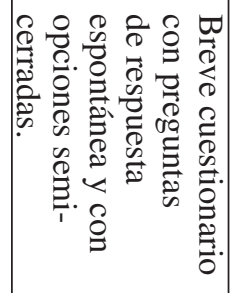 & 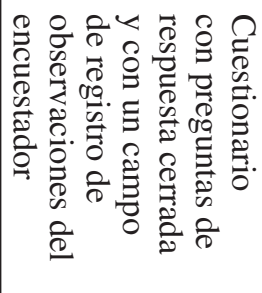 & 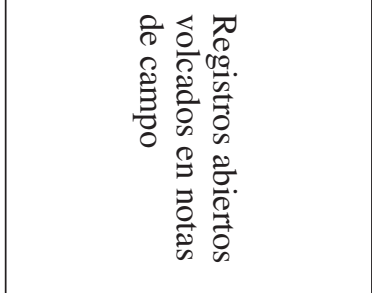 & 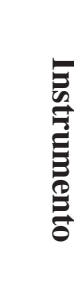 \\
\hline
\end{tabular}

EMPIRIA. Revista de Metodología de Ciencias Sociales. N. ${ }^{\circ}$ 34, mayo-agosto, 2016, pp. 15-38. ISSN: 1139-5737, DOI/empiria. 34.2016.16521 
La primera fase, "pre-mapeo" y "mapeo" buscó dar cuenta de la totalidad de lugares en el espacio público donde la práctica laboral discurre, así como observar, relevar, contabilizar el volumen y las características observables del trabajo infantil/adolescente/juvenil y de los sujetos que lo realizan, como insumo sustantivo para el cartografiado social del fenómeno, la sensibilización sobre los interrogantes e hipótesis, así como para la generación de un marco muestral de referencia para la fase 2 .

En la línea de los planteos de la cartografía social, se entiende que la espacialización de distintos procesos sociales habilita nuevas preguntas y reflexiones acerca del espacio y los usos de la ciudad. La decisión por la elaboración de un cartografiado respondió a entender al mapeo como una práctica en la cual el mapa funciona como herramienta para profundizar en la problematización de territorios sociales y los modos de habitarlos. Por esto mismo se cree necesario acompañar esta instancia con otras técnicas y soportes, porque a la imagen estática de un mapa hay que introducirle las subjetividades de quienes con sus prácticas construyen cotidianamente estos cartografiados que se superponen y muchas veces tensionan al mapa oficial que establece circuitos y usos predeterminados del espacio (Reyes 2008). Hay usos del espacio público que dan cuenta de disputas y relaciones de poder que es posible que sean observables, en nuestro caso, en circuitos y trayectorias de los niños/as, adolescentes y jóvenes que trabajan en la calle. El cartografiado da cuenta de una foto del momento en que se realizó, pero no repone de manera íntegra la subjetividad de los procesos territoriales. Si bien en esta investigación la construcción del mapa no tiene las características de una articulación colaborativa colectiva en los términos en que lo propone la corriente iconoclasista (Risler y Ares, 2013), esta imagen estática es un paso necesario no sólo como marco muestral y herramienta de diagnóstico de la situación que se aborda, sino como representación de usos de la ciudad para los que esos espacios no fueron pensados. Así, acompañar el mapa con un abordaje cualitativo y flexible en la segunda fase del trabajo de campo, responde a la necesidad de reponer las subjetividades.

En cuanto a la producción de la información, en la primera fase se utilizaron dos tipos de estrategias. Por un lado, para el "pre-mapeo" se trabajó desde una lógica flexible, en la que durante dos semanas se recorrieron distintos puntos de la ciudad, céntricos y periféricos, en diferentes horarios del día (mañana, tarde y noche) para observar los tipos de actividades que se desarrollaban y sus dinámicas. La información se recuperó a partir de observaciones y conversaciones informales con los propios trabajadores -niños/as, adolescentes y jóvenes- y con empleados de comercios, restaurantes, bares y cuidadores de parques, quienes adelantaron algunas pautas a considerar en el relevamiento (momentos de mayor intensidad del trabajo, modos de organización, etc.). Esta instancia cualitativa de la investigación, centrada en la observación y las entrevistas informales permitió ajustar los instrumentos y algunas de las decisiones sobre la operatoria del trabajo de campo posterior -mapeo-.

Por otro lado, para el mapeo, se construyó un instrumento destinado a 
detectar los casos de trabajo callejero y recuperar situaciones de personas sin techo ${ }^{6}$. En este instrumento se incluyeron indicadores que respondían a las siguientes dimensiones: contexto en el que se emplaza la actividad (zona, calle, turno de trabajo); características socio-demográficas del trabajador (sexo, edad, etc.); características del trabajo (grupal o individual, en punto fijo o en movimiento, tipo de trabajo (limosneo, venta de productos, etc.); uso de instrumentos/herramientas, lugar en el que pernocta (vivienda o espacio público) etc.; a su vez la planilla destinaba un espacio al registro abierto de observaciones del encuestador sobre el trabajador, sobre las características de la interacción tanto entre trabajadores como con el encuestador, etc. La finalidad de este instrumento, sencillo y muy simple de completar fue la de dimensionar de manera rápida y exhaustiva los casos existentes y algunos de sus atributos básicos.

En el marco de esta estrategia de relevamiento, el primer desafío metodológico fue el reconocimiento de los sujetos buscados. Si bien el foco en la actividad laboral hace que esta población resulte visible, delimitar el relevamiento a los niños/as, adolescentes y jóvenes representa una dificultad en tanto los límites de la observación impiden captar las edades. Si bien esto vale para todas las fronteras etarias, la primera dificultad de la fase 1 fue cómo reconocer a aquellos que forman parte de la población de estudio en términos generales, es decir, quiénes tienen menos de 25 años. Se buscó un acercamiento no invasivo a los sujetos generando conversaciones informales breves, que en algunos casos se acompañaban con la compra de algo de lo que ofrecían -fuesen objetos o servicios-. En general el acercamiento fue simple, y los sujetos no mostraron resistencias a la conversación y las preguntas, a excepción de unas pocas situaciones en las que claramente se advirtió cierta reactividad. En estos casos, se procedía al llenado del instrumento con los datos puramente observables, dejando vacías las celdas relativas a la información proporcionada por el sujeto. ${ }^{7}$ Por último, cabe agregar que aunque la definición operativa de trabajo infantil aquí considerada tomó como piso mínimo de edad era los 5 años,

${ }^{6}$ Cabe señalar que el registro de la situación de aquellos niños/as, adolescentes y jóvenes que se encuentran durmiendo en la calle responde al supuesto según el cual entendemos que para la reproducción de su vida cotidiana-sea porque viven en ella permanente o intermitentementerecurren a la realización de actividades generadoras de ingresos en el campo del trabajo informal en el espacio público.

7 Reflexionar sobre todo lo que conllevó el proceso de interacción entre investigador-investigado merecería un desarrollo particular aparte. Sí vale la pena adelantar que en el transcurso del trabajo de campo se comunicó a los sujetos sobre las intenciones y objetivos del estudio como así también se garantizó el anonimato y la confidencialidad de sus respuestas. En ocasiones se generaron tensiones, fundamentalmente con los niños de menor edad cuando se encontraban acompañados de adultos o con algunos niños y jóvenes que se encontraban notoriamente bajo los efectos de sustancias psicoactivas. En tales casos, se dejó a la libre elección del adulto la decisión al respecto de participar o no en el relevamiento. En todos los casos la participación en la investigación fue negociada, nunca impuesta, y cuando los niños se hallaban junto a algún adulto responsable se les consultaba primero a ello si podían participar de la investigación, luego, se hacía lo mismo con el niño. . 
igualmente y pese a las dificultades que ello plantea, se procedió a contabilizar a los niños/as que estuvieran por debajo de esa edad, en tanto se consideró necesario atender a esta situación de temprana experiencia en la calle.

El reconocimiento y la recolección necesariamente debieron tener en cuenta otra serie de particularidades de esta población. En primer lugar la movilidad, debido a que el desarrollo de las actividades laborales implica diversos patrones de uso y desplazamiento por el espacio urbano: utilización de puntos fijos que pueden variar luego de un período de tiempo, rotación o itinerancia permanente, circuitos establecidos y circuitos improvisados, etc., lo cual implica un desafío para el establecimiento de los espacios de observación. En segundo lugar, este tipo de actividades se desarrolla en distintos momentos del día y presenta variaciones estacionales, también derivadas de fenómenos climáticos (lluvias, vientos, temperaturas extremas) y del acceso a los productos que se pueden conseguir y vender, etc. Finalmente, la población presenta una alta rotación debido a sus características de actividad informal o no registrada, lo que se refleja en la dificultad metodológica de su captación específica en las encuestas oficiales sobre empleo y tiene como resultado la falta de un marco de muestreo.

Se capacitó a un grupo de observadores/encuestadores -quienes previo a la salida a campo probaron el instrumento-; se segmentó la totalidad del casco urbano en zonas -dado que el relevamiento tuvo un carácter censal - y se procedió a un conteo directo en dos momentos (mañana/tarde y noche, días de semana y fin de semana).

A continuación se muestra el mapa del casco urbano de la ciudad, cuya delimitación está fijada por las Avenidas 32, 120, 72 y 31. 


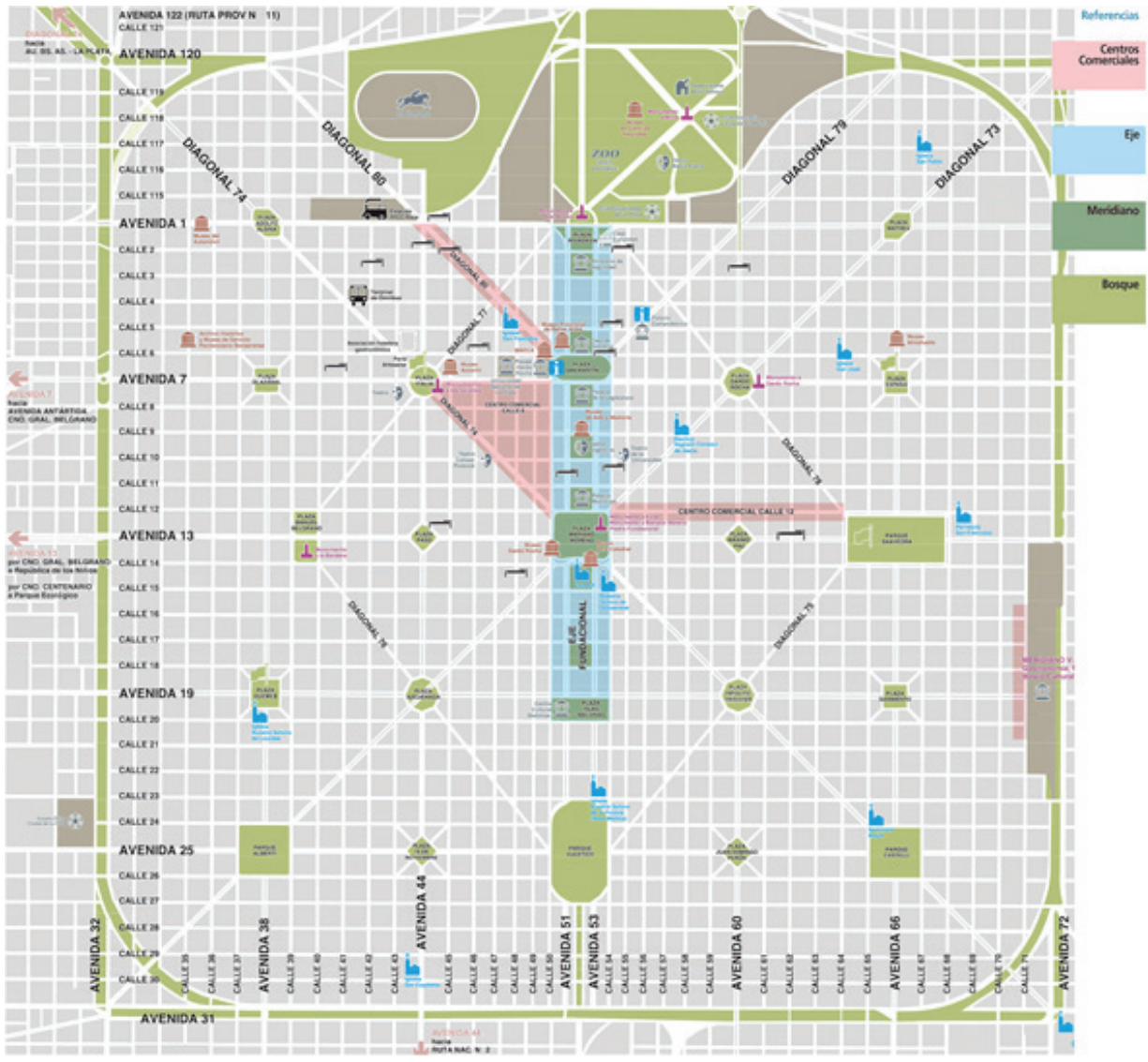

La estrategia de recorrido mutó según se tratase de zonas densamente pobladas de trabajadores (como el centro de la ciudad y alrededores) o escasamente pobladas. En el primer caso el recorrido se hizo a pie; en el segundo caso en automóviles. Además de registrar los puntos de trabajo fijo, se detectaron aquellas situaciones de trabajo móvil llevado a cabo en puntos estratégicos (circuitos de bares, restaurantes, edificios de la administración pública, etc.).

El relevamiento se realizó en noviembre -primavera-, durante dos miércoles y dos sábados consecutivos ${ }^{8}$. En cada uno de estos días se trabajó en dos

${ }^{8}$ El trabajo callejero tiene un carácter marcadamente estacional. Se eligió la primavera por ser un momento en el que comienza a activarse de manera considerable este tipo de inserciones laborales. Para que hubiera fidelidad en el registro se excluyeron días festivos (día de la madre, de la primavera, del maestro, etc.) por ser justamente los que aumentan el volumen de trabajadores pero de manera circunstancial. La elección de los días de la semana en que se efectuó el relevamiento tampoco fue azarosa. Al llevar a cabo el pre-mapeo se advirtió que el comportamiento del 
turnos: mañana/mediodía (de 11 a 16 hs.), y noche (de 20:30 en adelante). Cada observador repitió el mismo recorrido durante los días del trabajo de campo. Para el relevamiento nocturno se organizó el recorrido en grupos de dos observadores, planteando en ciertos segmentos de la ciudad la fijación -por momentos- del observador en determinados puntos fijos a fin de captar el trabajo móvil, muy característico en la nocturnidad.

Una serie de mecanismos implementados fueron efectivos para evitar las dificultades derivadas de un posible "doble conteo" de la población": 1) la simultaneidad del relevamiento en distintos puntos clave de la ciudad. 2) La máxima atención por parte de los observadores a fin de registrar las especificidades de cada trabajador (rasgos físicos, vestimenta, etc.). 3) La puesta en común de lo observado durante las reuniones que el equipo efectuó tras finalizar cada jornada de trabajo de campo. Allí se comentaron todos los casos identificados como "trabajo móvil" y aquellos de tipo "itinerante" -fijos en ciertos lugares determinados días, con traslados hacia otros lugares en otros días-; como así también se hizo un cruce de la información entre los encuestadores cuyas zonas eran colindantes. Se cree que la rigurosidad con la que se aplicaron estos mecanismos, permitieron un efectivo control del proceso.

Los resultados producidos en la primera fase, además de permitir el conteo global de las unidades de análisis y la caracterización genérica del fenómeno, fungieron de base sustantiva para pensar y repensar las decisiones de selección y recolección planteadas para la segunda fase, de cara a la profundización de los interrogantes sostenidos desde el inicio de la investigación. El procesamiento y análisis de los datos del censo permitió elaborar distintos perfiles de niños/ as, adolescentes y jóvenes trabajadores; en la construcción de los mismos se consideraron las variables edad, sexo y tipo de trabajo. Finalmente se configuró una muestra proporcional de estos perfiles, hacia la que se orientó el trabajo de campo de la fase 2.

En lo que respecta a esta segunda fase, se utilizaron dos técnicas integradas en un mismo instrumento y aplicadas en el mismo momento: una breve encuesta y una entrevista basada en un guión, ambas administradas a todos los sujetos que integraron la muestra. Para la primera se diseñó un cuestionario con preguntas de carácter espontáneo, con respuesta sobre opciones semi-cerradas orientadas a captar lugar de procedencia; asistencia escolar y nivel educativo alcanzado;

fenómeno variaba según se tratase de días laborables o días no laborables, de allí que se escogió un día típico de la semana, el miércoles, por estar justamente en el medio del transcurso de la misma, y un día típico de fin de semana, el sábado

9 En principio se pensó en aplicar para el conteo final el método "Capture / Recapture Analysis" -modelo originalmente empleado para estimar el tamaño de las poblaciones de vida silvestre- y que se ha aplicado a las poblaciones de humanos móviles, como los trabajadores agrícolas migrantes. El método requiere al menos dos observaciones independientes de la misma población. Con el fin de modelar el tamaño de la población $\mathrm{N}$, es necesario conocer: n, el número de miembros de la población observada por primera vez; $\mathrm{m}$, el número observado el segundo tiempo; y M, el número observado en ambas ocasiones. $\mathrm{N}$ se calcula entonces por $(\mathrm{n} * \mathrm{~m}) / \mathrm{M}$. Cada persona debe ser identificada con el fin de establecer M. Sin embargo, los mecanismos descriptos fueron suficientes, desestimando su aplicación. 
intensidad de la jornada laboral -cantidad de días a la semana y horas diarias promedio-; ingreso diario promedio. Esta búsqueda del conjunto de indicadores se aplicó en articulación con el dispositivo de entrevista, por ello el carácter espontáneo de las respuestas más cercano a un tipo de indagación cualitativo.

Para la realización de las entrevistas se diseñó un guión flexible, que si bien priorizó la profundización de la dimensión laboral (inicios en el mundo del trabajo; experiencias anteriores; organización de la actividad actual -entrada, vínculos con otros trabajadores callejeros, con peatones, con la policía, provisión de instrumentos de trabajo y productos, administración del tiempo, del dinero, gestión del espacio de trabajo, etc.-; representaciones sobre el trabajo, la calle y el trabajo callejero; expectativas a futuro), también ahondó en otras esferas de la trayectoria biográfica tales como: educación; sociabilidad; vínculos familiares, ocio y recepción de políticas públicas.

Al igual que en la fase 1, en esta segunda fase, el trabajo de campo se desarrolló durante la primavera, en los meses de noviembre y diciembre del año siguiente al de la realización del mapeo. En esta instancia participó el mismo equipo, que fue nuevamente capacitado, esta vez, en las especificidades y sensibilidades que un abordaje cualitativo con población de características complejas requiere. El énfasis de las entrevistas estuvo puesto es la flexibilidad y la capacidad de captar temáticas emergentes no anticipadas en el guión.

Esta etapa concluyó y el equipo se encuentra actualmente trabajando en el análisis de los datos producidos en la segunda fase.

\section{REFLEXIONES FINALES}

Este artículo se planteó como objetivo central explicitar un conjunto de decisiones teórico-metodológicas puestas en juego para el estudio in situ de las actividades laborales llevadas a cabo por niños/as, adolescentes y jóvenes en el espacio público de la Ciudad de La Plata (Buenos Aires, Argentina). Para ello:

1) Se presentó la delimitación del problema que abordó la investigación empírica llevada a cabo, como así también se explicitó el núcleo de preguntas cognitivas que la orientaron.

2) Se plantearon las decisiones teóricas y los recortes conceptuales de las nociones estructurantes para el estudio en cuestión. Al respecto de esto último se recuperó la discusión en torno a la ya clásica aproximación "chicos en/de la calle", reemplazada por la noción de chicos/as que trabajan en la calle; luego se avanzó en la delimitación de las categorías de trabajo infantil, adolescente y juvenil, para por último definir teórica y operativamente el concepto de espacio público.

3) Se explicitaron las decisiones metodológicas de todo el proceso supeditadas al problema de investigación formulado- lo cual implicó una primera definición: la opción por un diseño mixto secuencial en dos fases (Teddlie y Tashakori, 2006). Luego, se desarrollaron las definiciones en torno a los procesos de a) selección (definición de las unidades de análisis, de los espacios 
de observación y construcción de la muestra) y b) recolección (tanto referida al diseño de los instrumentos como a la definición de aspectos operativos y realización del trabajo de campo).

En suma, esta explicitación buscó dar cuenta de lo que en el ámbito de la metodología se conoce como la "trastienda de la investigación" (Wainerman, 2001). Asumiendo que una buena parte de las investigaciones de este campo de estudios se concentra en la comunicación de resultados y suele dejar poco espacio para la problematización de la construcción del dato, en esta comunicación se buscó dar cuenta de la trama de decisiones que este proceso implica. Como se explicó en la introducción, se considera que la explicitación de tales decisiones puede aportar dos insumos: 1) el modo en que se construye sociológicamente el abordaje de un objeto de investigación complejo -desde la formulación inicial de las preguntas, su justificación y la delimitación de las definiciones teóricas y operativas; y 2) la forma en que se tomaron las decisiones metodológicas en un abordaje basado en un enfoque mixto. Ambas cuestiones devienen en herramientas concretas y de utilidad para quienes procuren desarrollar una investigación de similares características

\section{BIBLIOGRAFÍA}

APTEKAR, L. y ABEBE, B. (1997): "Conflict in the neiborhood: street and working children in the public space", Childhood. A global Journal of child research, 4 (4), pp. 477-490.

BECKER, H. (2009): Trucos del oficio. Cómo conducir su investigación en ciencias sociales, Buenos Aires, Siglo XXI.

BURT, M. R., ARON LAUDAN, Y., DOUGLAS T., VALENTE, J., LEE, E., IWEN B.(1999): Homelessness: Programs and the People they Serve. Findings of the National Survey of Homeless Assistance Providers and Clients.Technical report, Washington, DC, Urban Institute Press, disponible en: http://www.huduser.org/ publications/homeless/homeless-tech.html [Consulta: 6/03/2014].

BOUFFARTIQUE, P. (1996): “¿Fin del trabajo o crisis del trabajo asalariado?”, Revista Sociología del Trabajo, 29, pp. 91-110.

CERQUIERA FILHO, G. y NEDER, G. (2001): "Social and historical approaches regarding street children in Rio de Janeiro (Brasil) in the context to the transition to democracy", Childhood. A global Journal of child research, 8 (1), pp. 11-29.

DALLAPE, F. (1996): "Urban Children: a challange and an opportunity", Childhood. A global Journal of child research, 3, pp. 283-294.

DE LA GARZA TOLEDO, E. (2001): "Problemas clásicos y actuales de la crisis del trabajo", El futuro del trabajo, el trabajo del futuro, México, CLACSO, pp. 11-32.

DENZIN, N. (1979): The research act: a theoretical introduction to sociological methods, New York, Mc Graw Hill.

DROZ, I. (2006): "Street Children and the work ethic. New policy for an old moral, Nairobi (Kenya)", Childhood. A global Journal of child research, 13 (3), pp. 349363. 
FIRDION, J. M., MARPSAT, M. y MAUGER, G. (2000): Les séminaires de la valorisation de la recherche: Etude des sans-domicile: Le cas de Paris et de l'Ile de France", Paris, INED.

FIRDION, J. M. y MARPSAT, M. (2007): "A Research Program on Homelessness in France", Journal of Social Issues, 63 (3), pp. 567-588.

FIRDION, J. M., MARPSAT, M. y MERON, M. (2000): "The difficult past of homeless young people", Population y societies, 363, pp- 1-4.

GARCÍA SILVA, R. (2014): Los chicos en la calle. Llegar, vivir y salir de la intemperie urbana, Buenos Aires, Espacio Editorial.

GIDDENS, A. (1998): La constitución de la sociedad. Bases para la teoría de la estructuración, Buenos Aires, Amorrortu.

GOFFMAN, E. (2006): La presentación de la persona en la vida cotidiana, Buenos Aires: Amorrortu.

GREGORI, M. F. (2000): Viracao: Experiencias de meninos nas ruas, San Pablo, Companía das Letras.

GROPPO, L. A. (2000): Juventude. Ensayos sobre Sociología e História das Juventudes Modernas, Río de Janeiro, DIFEL.

HETCH, T. (1998): At home in the street. Street children of northeast Brasil, United Kindom, Cambridge University Press.

HUGGINGS, M. y RODRIGUES, S. (2004): "Kids working on Paulista Avenue", Childhood. A global Journal of child research, 11 (4), pp. 495-514.

INVERNIZZI, A. (2003): "Street working children and adolescents in Lima. Works as an agent of socialization", Childhood. A global Journal of child research., 10 (3), pp. 319-341.

KESSLER, G. (2006): Sociología del delito amateur, Buenos Aires, Paidós.

KOHEN, J. (2004): La problemática del trabajo infantil y docente en el contexto de las nuevas vulnerabilidades. Del impacto negativo en la salud a la búsqueda de procesos saludables, Tesis Doctoral (Publicada por la Universidad Nacional de Rosario).

LEYRA, B. (2009): Trabajo infantil femenino: niñas trabajadoras en ciudad de México, Tesis Doctoral, Universidad Complutense de Madrid.

LINDÓN, A. (1997): "El trabajo y la vida cotidiana. Un enfoque desde los espacios de vida", Economía, Sociedad y Territorio, 1 (1), pp. 176-197.

LINDÓN, A. (2002): "Trabajo, espacios de vida y cotidianeidad. La periferia oriental de la ciudad de México", Scripta Nova. Revista electrónica de geografía y ciencias sociales, VI (119), pp. 1-19.

LINDÓN, A. (2008): "Los giros de la geografía urbana: frente a la pantópolis, la microgeografía urbana", Ponencia presentada en el X Coloquio Internacional de Geocrítica. Barcelona 26-20 de mayo de 2008.

LUCCINI, R. (1996): "The street and its image", Childhood. A global Journal of child research, 3, pp. 235-243.

MAKOWSKI, S. (2010): Jóvenes que viven en la calle, México, Siglo XXI.

MAKOWSKI, S. y FLORES, J. (2010): Niños, niñas, adolescentes y jóvenes en situaciones de calle. Elementos para repensar las formas de intervención, México, Lenguaraz.

MARPSAT, M. y FIRDION, J. M. (1998): "Las personas sin hogar en París: encuesta a una muestra representative de usuarios de servicios para personas sin hogar", Intervención psicosocial, 7 (1), pp. 47-63.

MARPSAT, M. y FIRDION, J. M (2001): "Les ressourses des jeunes sans domicile et en situation precarie", Recherches et previsions, 65, pp. 91-112. 
MARPSAT, M. (2008a): “L'enquête de l'Insee sur les sans-domicile: quelques éléments historiques", Courrier des statistiques, 123, pp. 53-64.

MARPSAT, M. (2008b): The INED research on homelessness, 1993-2008, Documents de travail, 1 (156). Paris, INED.

MíGUEZ, D. (2008): Delito y cultura. Los códigos de la ilegalidad en la juventud marginal urbana, Buenos Aires, Biblos.

MINISTERIO DE TRABAJO, EMPLEO Y SEGURIDAD SOCIAL (2014): Situación del trabajo infantil en el total urbano nacional. Adelanto del Informe de resultados del Módulo de Actividades de Niños, Niñas y Adolescentes, Buenos Aires, MTEySS y OIT.

OIT (2002): "Romania Working Street children in Bucharest: a rapid assessment", en Serie: Investigating the worst forms of child labour, 19, Ginebra, OIT.

OIT (2011): Child labour in Papua New Guinea. Report on the rapid assessment in Port Moresby in commercial sexual exploitation of children and children workimg on the streets, Fiji, OIT.

PAUGAM, S. (2007): Sortir de la rue. Les sans abri. Conference de consensus. Synthèse des études sur les dimensions sociologique et anthropologique du phénomène: facteurs explicatifs, modes de vie et trajectoires de sorti, Paris, 29 al 30 de noviembre.

PAWSON, R. (1994): «Quality and Quantity, Agency and Structure, Mechanism and Context, Dons and Cons», Nuffield College 1993/ World Congress of Sociology, ISA, Bielefeld M/S.

PEIRÓ, M. L. y RAUSKY, M. E. (2009): "Los organismos internacionales frente al trabajo infantil y juvenil: aportes para un análisis de sus discursos y propuestas", Cuestiones de Sociología. Revista de Estudios Sociales, 5/6, pp. 313-338.

PERALTA, M.I, AMMAN, B., ANDRADA, S., FAAS, A., MUCHIUT, M., MARASCA. M., MARASCA, R., AHUMADA, M.I. (2011): Censo de niños, niñas y adolescentes en situación de calle, Córdoba, Universidad Nacional de Córdoba.

PERGAMIT, M., CUNNINGHAM, M., BURT, M., LEE, P., HOWELL, B., y BERTUMEN, K. (2013): Youth Count! Process study, Urban Institute, EE.UU.

PÉREZ ALVAREZ, A. (2003): Maniobras de la sobrevivencia en la ciudad. Territorios de trabajo informal infantil y juvenil en espacios públicos del centro de MedellínColombia, Tesis de grado, Colombia.

PÉREZ LÓPEZ, R. (s/f): Los niños de la calle: aprendiendo a sobrevivir: El uso del comercio informal como estrategia de subsistencia para los niños y jóvenes en la ciudad de México, Memoria del seminario El ambulantaje en la ciudad de México. Investigaciones recientes, México, UNAM.

POJOMOVSKY, J. (2008): Cruzar la calle. Niñez y adolescencia en las calles de la Ciudad, Buenos Aires, Biblos.

REYES, D. y FUENTES, M. (2008): Metodología de cartografía participativa y sistematización de información espacial. Secretaría distrital de integración social, Alcaldía Mayor de Bogotá DC.

RIAL, V., RODRIGUEZ , E., Y VOMERO, F. (s/f): Varones jóvenes en situación de calle. Entre el estigma y la marginalidad

RISLER, J. Y ARES, P. (2013): Manual de mapeo colectivo. Recursos cartográficos críticos para procesos territoriales de creación colaborativa, Buenos Aires, Tinta limón.

RIZZINI, I. (1996): "Street children: an excluded generation in Latin América", Childhood. A global Journal of child research, 3, pp. 215-233. 
RIZZINI, I., CALDEIRA, P., RIBEIRO, R., y CARVANO, L. M. (2010): Criancas e adolescentes com direitos violados. Situacao de rua e indicadores de vulnerabilidade no Brasil urbano, Cuadernos, 2, Río de Janeiro, CIESPI-PUC RIO.

SCHEPER-HUGHES, N. y HOFFMAN, D. (1999): "Brazilian Apartheid: street kids and struggle for urban space", en Small Wars. The cultural politics of childhood. Berkeley, University of California Press, pp- 352-389.

TEDDLIE, A. y TASHAKORI, Ch. (2005): "Mixed methods research tradition", en Encyclopedia of Educational Administration, Thousand Oaks, CA, Sage.

UNICEF (2000): Estudio de niños, niñas y jóvenes trabajadores en el Distrito Federal. México, UNICEF.

WAINERMAN, C. y SAUTU, R. (2001): La trastienda de la investigación. Buenos Aires, Lumiere. 